\title{
Observation of Nonspreading Wave Packets in an Imaginary Potential
}

\author{
R. Stützle* M.C. Göbel, Th. Hörner, E. Kierig, I. Mourachko, and M.K. Oberthaler \\ Kirchhoff-Institut für Physik, Universität Heidelberg, \\ Im Neuenheimer Feld 227, D-69120 Heidelberg, Germand
}

\author{
M.A. Efremov, ${ }^{1}$ M.V. Fedorov,${ }^{1}$ V.P. Yakovlev, ${ }^{2}$ K.A.H. van Leeuwen, ${ }^{3}$ and W.P. Schleich ${ }^{4}$ \\ ${ }^{1}$ General Physics Institute, Russian Academy of Sciences, 38 Vavilov Street, Moscow, 119991 Russia \\ ${ }^{2}$ Moscow Engineering Physics Institute (State University), 31 Kashirskoe shosse, Moscow, 115409 Russia \\ ${ }^{3}$ Eindhoven University of Technology, P.O. Box 513, 5600 MB Eindhoven, The Netherlands and \\ ${ }^{4}$ Abteilung für Quantenphysik, Universität Ulm, D-89069 Ulm, Germany
}

(Dated: October 3, 2018)

\begin{abstract}
We propose and experimentally demonstrate a method to prepare a nonspreading atomic wave packet. Our technique relies on a spatially modulated absorption constantly chiseling away from an initially broad de Broglie wave. The resulting contraction is balanced by dispersion due to Heisenberg's uncertainty principle. This quantum evolution results in the formation of a nonspreading wave packet of Gaussian form with a spatially quadratic phase. Experimentally, we confirm these predictions by observing the evolution of the momentum distribution. Moreover, by employing interferometric techniques, we measure the predicted quadratic phase across the wave packet. Nonspreading wave packets of this kind also exist in two space dimensions and we can control their amplitude and phase using optical elements.
\end{abstract}

PACS numbers: 03.75.Be, 42.50.Vk, 03.75.Dg

Nonspreading wave packets have attracted interest since the early days of quantum mechanics. Already in 1926 Schrödinger [1] found that the displaced Gaussian ground state of a harmonic oscillator experiences conformal evolution because a classical force prevents the wave packet from spreading. Even in free space the correlations between position and momentum stored in an initially Airy-function-shaped wave packet can prevent spreading 2]. Here we propose and experimentally observe the formation and propagation of nondispersive atomic wave packets in an imaginary (absorptive) potential accessible in atom optics [3, 4, [5]. Although there is no classical force, there are correlations continuously imposed by Heisenberg's uncertainty relation resulting in the stabilization of the wave packet.

Localized wave packets due to stabilization are well known in the context of periodically driven quantum systems [6] and studied with increasing interest for electronic wave packets in Rydberg atoms 7, 8, 9, 10. Our approach to create nondispersive atomic wave packets relies on three ingredients: (i) an absorption process [1] cuts away the unwanted parts of a broad wave creating a packet that is continuously contracting in position space, (ii) this process leads due to Heisenberg's uncertainty relation to a broadening in momentum space and consequently to a faster spreading in real space, and (iii) the absorptive narrowing and the quantum spreading are balanced, leading to a nonspreading wave packet. In the following we will refer to such a wave packet as Michelangelo packet [12].

Complex potentials for matter waves [13] emerge from the interaction of near resonant light with an open twolevel system shown in Fig. I(a). For a standing light wave tuned exactly on resonance an array of purely imaginary harmonic potentials arises. When the Rabi frequency $\Omega_{0}$ is of the order of the excited state linewidth $\Gamma$ the local saturation parameter $\left|\Omega_{0} \sin (k x) / \Gamma\right|$, and thus the upper level population, is of the order of unity except in a small vicinity of the field nodes. Consequently, our system decays approximately with the rate $\Gamma$. Therefore, in the time domain $t \gg 1 / \Gamma$ the atomic wave function vanishes almost everywhere, except in small vicinity $\delta x$ of the field nodes. Here the saturation is small and our open system decays with the rate $\left(\Omega_{0} k \delta x\right)^{2} / \Gamma \ll \Gamma$. We estimate the time dependent size $\delta x$ from the relation $\left(\Omega_{0} k \delta x\right)^{2} t / \Gamma \sim 1$ and find $\delta x(t) \sim(\Gamma / t)^{1 / 2} /\left(k \Omega_{0}\right)$.

The decrease of $\delta x(t)$ is accompanied by an increase of the width $\delta p(t) \sim 1 / \delta x(t)$ in momentum space [14] leading to spatial spreading of the wave packet. Because of competition of the two processes - absorptive contraction and quantum spreading - the width $\delta x(t)$ reaches its minimal stationary value $\delta x_{0}$. In this asymptotic regime the rate $\delta x(t) / t$ of absorptive contraction is obviously balanced by the rate $\delta p(t) / M$ of quantum spreading which yields the characteristic time $t_{0} \equiv 1 / \omega_{0} \sim \Omega_{0}^{-1}\left(\Gamma / \omega_{r}\right)^{1 / 2}$ and the stationary width $\delta x_{0} \sim\left(M \omega_{0}\right)^{-1 / 2}$ with the recoil frequency $\omega_{r} \equiv k^{2} /(2 M) \ll \Gamma$.

The experiments are performed with a slow atomic beam of metastable argon $(v=50 \mathrm{~m} / \mathrm{s})$ produced with a standard Zeeman slower. The brilliance of the beam is significantly enhanced with a 2D-MOT setup [15]. The final collimation necessary for coherent illumination is obtained by two slits $(25 \mu \mathrm{m}$ and $10 \mu \mathrm{m})$ within a distance of $25 \mathrm{~cm}$. Applying a Stern-Gerlach magnetic field we select the atoms in the internal state $1 s_{5}(J=2$, $\left.m_{j}=0\right)$. The imaginary potential is realized with a 


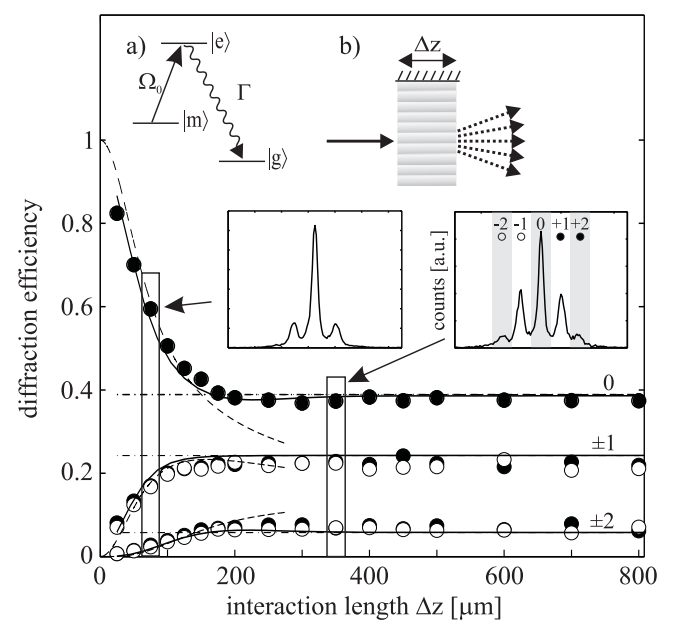

FIG. 1: Formation of a nonspreading Michelangelo wave packet for the center-of-mass motion of an open two-level atom (a). The resonant interaction with a standing light wave (b) leads to an array of harmonic imaginary potentials. The normalized diffraction efficiencies derived from the momentum distributions (inset) approach a steady state as a function of the interaction length $\Delta z$ demonstrating the successful realization of stationary wave packets. The solid curves result from a numerical integration of the Schrödinger equation [16] with the Rabi frequency $\Omega_{0}=0.4 \Gamma$. The dashed lines correspond to the Raman-Nath approximation, revealing that the interplay between absorption and quantum spreading is essential for obtaining a steady state.

circularly polarized standing light wave by retroreflecting a laser beam resonant with the $1 s_{5}-2 p_{8}$ transition $(801 \mathrm{~nm})$. This setup realizes to a very good approximation an open two-level system since only $16 \%$ of the excited atoms fall back to the initial state (in contrast to $32 \%$ without magnetic state selection). In order to control the interaction length $\Delta z$, the laser beam passes an adjustable slit. By imaging the slit onto the retroreflecting mirror we avoid the spoiling effect of light diffraction. The detection of the metastable argon atoms is achieved by a microchannel plate detector allowing for spatially resolved single atom detection utilizing their internal energy $(12 \mathrm{eV})$. Since the transverse coherence length of the incoming atomic beam is much larger than the optical wave length, the outgoing wave function is a coherent array of single Michelangelo wave packets, resulting in constructive interference in certain directions. The spatial resolution $\sim 50 \mu \mathrm{m}$ of our atomic detector and the free flight distance $\sim 0.5 \mathrm{~m}$ guarantee clearly resolving the resulting atomic diffraction pattern in the far field.

The diffraction efficiency is deduced by summing up the detected number of atoms in angular windows as indicated in the right inset of Fig. 1 After their initial dynamics the wave packets, i.e., the diffraction efficiencies do not change giving evidence to the formation of Michelangelo wave packets [17]. Our numerical simulations (solid line) of the open two-level Schrödinger equation take into account the longitudinal as well as the transverse velocity distributions $\Delta v_{l}=10 \mathrm{~m} / \mathrm{s}$ and $\Delta v_{t}=7 \mathrm{~mm} / \mathrm{s}$ of the experiment. For $\Omega_{0}=0.4 \Gamma$ we have a very good agreement with our experimental findings. Since this agreement depends critically on the Rabi frequency we can determine its absolute value. It is consistent within a factor of 2 both with a rough estimate, using the power measurement of the incoming light beam, and with the overall absorption of the atomic beam.

In order to stress that the interplay between absorptive narrowing and the quantum spreading is crucial for the formation of the Michelangelo packet, we have included the result of the Raman-Nath approximation (dashed lines). Since this approach is only valid as long as quantum spreading is negligible, it fails to predict the resulting dynamics after the characteristic time $t_{0}$.

According to the arguments given above, Michelangelo wave packets emerge after a characteristic time $t_{0} \sim 1 / \Omega_{0}$. Our experimental results shown in Fig. 2 confirm the expected scaling with $\Omega_{\min }=0.23 \Gamma$.

We now show that a Michelangelo wave packet is a complex Gaussian wave packet with a quadratic phase. For this purpose we recall [16] that the solution of the Schrödinger equation

$$
i \frac{\partial}{\partial t} \varphi(x, t)=\left(-\frac{1}{2 M} \frac{\partial^{2}}{\partial x^{2}}-i U_{2}(x)\right) \varphi(x, t)
$$

for the metastable state wave function $\varphi(x, t)$ in the vicinity of $x=0$, where $U_{2}(x)=M \omega_{0}^{2} x^{2} / 2$ with $\omega_{0} \equiv$ $\Omega_{0} \sqrt{2 \omega_{r} / \Gamma}$ reads [5]

$$
\varphi(x, t)=\sqrt{\frac{k / \pi}{\cosh \beta t}} \exp \left(-\frac{1}{2} \alpha x^{2} \tanh \beta t\right),
$$

with $\alpha \equiv M \omega_{0} \exp (-i \pi / 4)$ and $\beta \equiv \omega_{0} \exp (i \pi / 4)$.

Hence, the probability density $|\varphi(x, t)|^{2}$ is a Gaussian with the time dependent width $\delta x(t) \equiv$ $[\operatorname{Re}\{\alpha \tanh (\beta t)\}]^{-1 / 2}$, which for $\omega_{0} t>1$ reaches its minimal stationary value $k \delta x_{0} \equiv\left(\omega_{r} \Gamma / \Omega_{0}^{2}\right)^{1 / 4}$.

In this asymptotic regime Eq. (2) factorizes into a product of the time dependent function $\cosh ^{-1 / 2}(\beta t)$, showing that the Michelangelo probability density decays exponentially in time with the rate $\Gamma_{0} \equiv \omega_{0} / \sqrt{2} \ll$ $\Gamma$, and the position dependent complex Gaussian $\exp \left(-\alpha x^{2} / 2\right)$ which contains the quadratic phase $\phi(x) \equiv$ $M \omega_{0} x^{2} / \sqrt{8}$. A Fourier transformation of this wave packet with the stationary width $\delta x_{0}$, yields the asymptotic behavior of the diffraction efficiencies shown in Fig. 1 by the dashed-dotted lines and is in perfect agreement with our experimental findings.

The predicted phase $\phi(x)$ of the Michelangelo packet can be deduced from the phases of the observed diffraction orders where the phase of the $n$th order with respect to the zeroth order is $\phi(n)=-2\left(\omega_{r} \Gamma / \Omega_{0}^{2}\right)^{1 / 2} n^{2} \equiv \phi_{2} n^{2}$. To measure the relative phases we realize a compact 


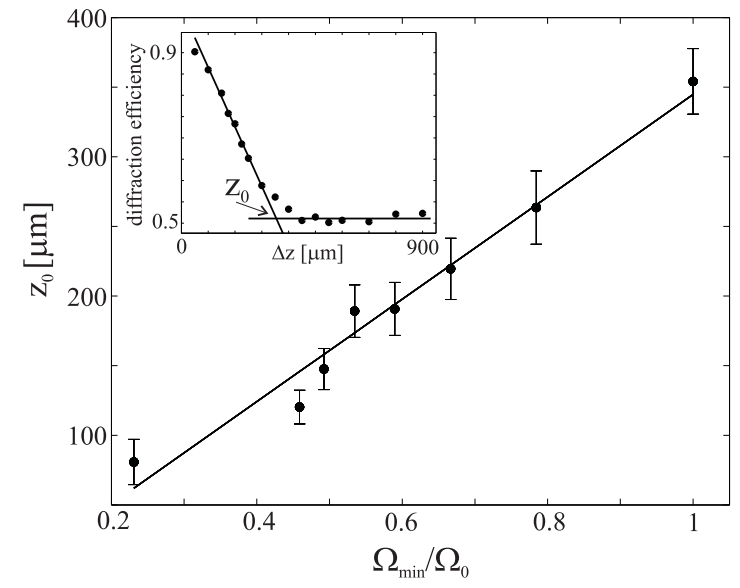

FIG. 2: Experimental verification of the scaling law $t_{0} \sim$ $1 / \Omega_{0}$ connecting the characteristic time $t_{0} \equiv z_{0} / v$ when Michelangelo wave packets form and the Rabi frequency $\Omega_{0}$. The line is a guide to the eye. We measure the zeroth order diffraction efficiency as a function of $\Delta z$ (inset) for different Rabi frequencies. The crossing point between the linear extrapolation of the short and long-time limits yields $z_{0}$.

interferometer setup shown in Fig. 33. A thin nearresonant probing standing light wave (waist $30 \mu \mathrm{m}$ ) is placed directly behind the array of harmonic imaginary potentials. The wave function amplitude in each output direction is given as a superposition of different diffraction orders of the Michelangelo packet. By changing the relative phase between the two standing light waves we can measure an interference pattern and thus deduce the phase evolution as a function of the interaction length $\Delta z$.

The interferometric setup employs a probing standing wave at $801 \mathrm{~nm}$ realized by beams impinging on the mirror under an angle of $10^{\circ}$. Thus, moving the mirror allows us to scan the relative phase $\phi_{s}$ between the probing and the absorptive light wave (beating period $25 \mu \mathrm{m}$ ). The presence of a magnetic field in the interaction region enables us to realize a detuned $(8 \mathrm{MHz})$ probing wave using the same laser for both standing light waves but different circular polarizations. By detuning the probing light wave the total flux through the interferometric setup is significantly increased in comparison to an exactly resonant probing field.

In order to deduce the absolute value of $\phi_{2}$ we evaluate the interferometer output in the direction of the third diffraction order. For our experimental parameters this beam is always a two-beam interference of the first and second diffraction order of the array of Michelangelo packets. In contrast, the output in lower diffraction order directions is the result of multiple-beam interference and does not allow us easily to deduce the involved phases.

In order to find the phase difference $\phi(2)-\phi(1)$ we have to eliminate the offset phase arising mainly from the fact that the probing light field is not infinitely thin.

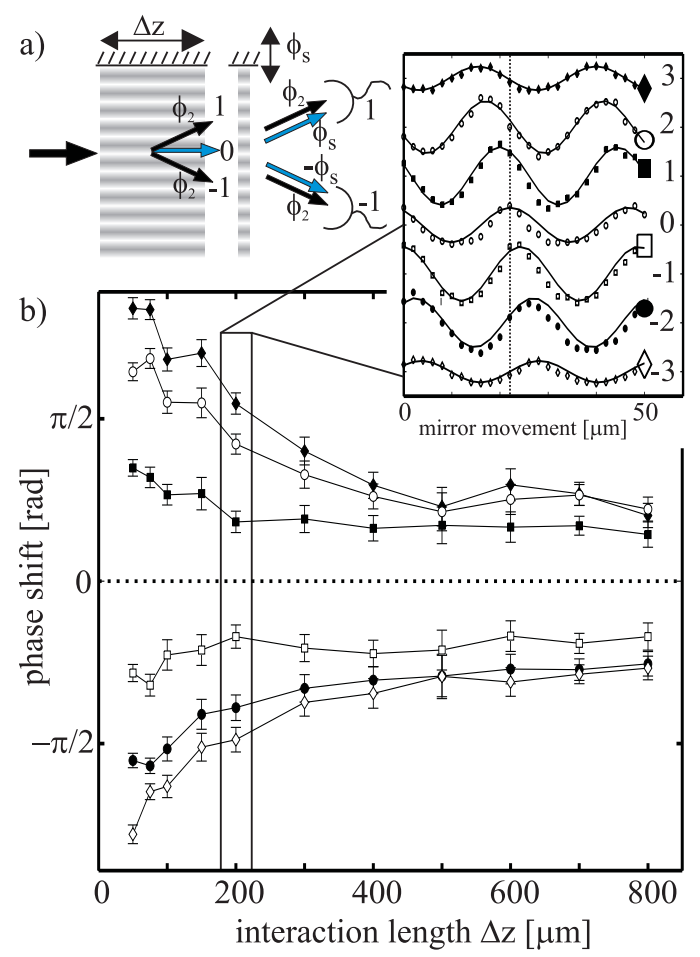

FIG. 3: Measurement of the phase of a Michelangelo wave packet using an interferometric setup (a) consisting of the absorptive and probing standing waves. The inset in (b) shows typical interference patterns for different output directions obtained by scanning the relative position of the second (thin) standing light wave for a given interaction length $\Delta z$. For large values of $\Delta z$ the phase shifts (b) of the different interferometer outputs relative to the zeroth order level off, indicating stationary phases of the wave packet.

For this purpose we take the difference between the measured phase in the long-time limit of the absorptive wave $(\Delta z>400 \mu \mathrm{m})$ and the phase for the experimentally achievable shortest interaction length $(50 \mu \mathrm{m})$. For the Rabi frequency $\Omega_{0}=(0.23 \pm 0.02) \Gamma$ we find the experimental value $|\phi(2)-\phi(1)|=1.70 \pm 0.17$, which is in agreement with the prediction of the numerical integration $\phi(2)-\phi(1)=3 \phi_{2}$, that is $\left|\phi_{2}\right|=0.57 \pm 0.1$. Moreover, the characteristic length $z_{0} \sim 400 \mu \mathrm{m}$ for leveling off the phases coincides with the one for leveling off the diffraction efficiencies. Furthermore, by increasing the Rabi frequency to $\Omega_{0}=(0.4 \pm 0.05) \Gamma$ we experimentally deduce $\left|\phi_{2}\right|=0.32 \pm 0.08$, which is in very good agreement with the prediction of the numerical integration $\left|\phi_{2}\right|=0.27 \pm 0.04$.

So far we have concentrated on wave packets in $D=1$ spatial dimensions. A straightforward generalization to $D=2$ relies on two orthorgonal linear polarized standing waves interacting with the appropriate atomic transitions and leads to the potential $-i M\left(\omega_{x}^{2} x^{2}+\omega_{y}^{2} y^{2}\right) / 2$ near the nodes. The frequencies $\omega_{x}$ and $\omega_{y}$ depend on the field intensities. A nonorthorgonal configuration provides even 


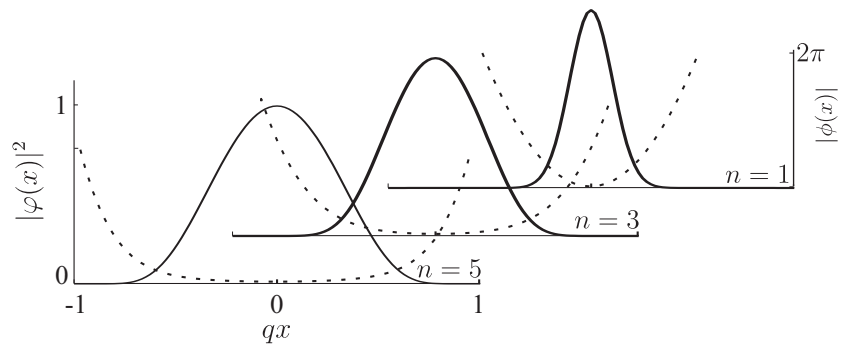

FIG. 4: Probability density $|\varphi(x)|^{2}$ (solid line) and absolute value of phase $|\phi(x)|$ (dotted line) of Michelangelo wave packets in the Potential $U_{2 n}$.

additional parameters to control the form of the emerging two-dimensional Michelangelo wave packet.

We emphasize that Michelangelo wave packets are not restricted to the Gaussian form, Eq. (2), originating from the quadratic potential $U_{2}$ in Eq. (11). Indeed, with an appropriate mask [18] we can create almost any behavior of the mode function close to the node, leading, for example, to a power law potential $U_{2 n}(x)=\left(\Omega_{0}^{2} / \Gamma\right)(q x)^{2 n}$. Here $q \ll k$ determines the characteristic width of $U_{2 n}$.

The Michelangelo wave packets shown in Fig. 4 for $n=1,3$, and 5 are the "ground" state eigenfunctions of the corresponding stationary non-Hermitian Hamiltonians and can be obtained numerically. In the asymptotic regime only these functions survive because their complex energy "eigenvalues" have the smallest imaginary parts. Moreover, applying the general arguments above to the case of $U_{2 n}$ yields the following characteristic time and width:

$t_{0} \sim \frac{1}{\Omega_{0}^{2 /(n+1)}}\left(\frac{\Gamma}{\tilde{\omega}_{r}^{n}}\right)^{1 /(n+1)}$ and $q \delta x_{0} \sim\left(\frac{\Gamma}{\Omega_{0}^{2} t_{0}}\right)^{1 / 2 n}$,

where $\tilde{\omega}_{r}=q^{2} /(2 M)$. These scaling behaviors have been confirmed by numerical integration of the corresponding Schrödinger equation. We note that for $n=1$ these expressions reduce to the ones of the previous case. For $n \rightarrow \infty$ the potential $U_{2 n}$ takes on the shape of a box, $t_{0}$ is independent of $\Omega_{0}$, and $\delta x_{0}$ is solely given by $q$.

In conclusion we present a new class of nonspreading wave packets resulting from the interplay between absorptive narrowing and quantum spreading. The developed theoretical description explains the experimental observation of both the phase and the amplitude of the wave packet quantitatively. The experimental realization of imaginary potentials strongly relies on spontaneous decay processes. Nevertheless, we show that coherence is maintained and can even be employed for deducing the phase of the Michelangelo packets. Since the wave packet arising in the long-time limit is weakly dependent on the initial wave function, this process is a robust tool for generating wave packets with well-defined amplitude and phase for further experiments.
We acknowledge fruitful discussions with A. Buchleitner and thank M. Störzer for his commitment in the early stage of the experiment which was funded by OptikZentrum Konstanz, Center for Junior Research Fellows in Konstanz, by Deutsche Forschungsgemeinschaft (Emmy Noether Program), and by the European Union, Contract No. HPRN-CT-2000-00125. MVF, WPS, and VPY also thank the Alexander von Humboldt-Stiftung for its generous support during the course of this project, especially for the Humboldt-Kolleg at Cuernavaca, Mexico. This work was partially supported by the Landesstiftung Baden-Württemberg and the Russian Foundation for Basic Research (grants no. 02-02-16400, 03-02-06145, 04-0216734).

* Electronic address: stuetzle@kip.uni-heidelberg.de

† URL: www.kip.uni-heidelberg.de/matterwaveoptics

[1] E. Schrödinger, Naturwissenschaften 14, 664 (1926).

[2] M.V. Berry and N.L. Balazs, Am. J. Phys. 47, 264 (1979).

[3] D.O. Chudesnikov and V.P. Yakovlev, Laser Phys. 1, 110 (1991).

[4] M.K. Oberthaler et al., Phys. Rev. Lett. 77, 4980 (1996).

[5] M.V. Berry and D.H.J. O’Dell, J. Phys. A 31, 2093 (1998).

[6] A. Buchleitner, D. Delande, and J. Zakrzewski, Phys. Rep. 368, 409 (2002), and references therein.

[7] G.P. Berman and G.M. Zaslavsky, Phys. Lett. A 61, 295 (1977); K. Richter and D. Wintgen, Phys. Rev. Lett. 65, 1965 (1990); J. Henkel and M. Holthaus, Phys. Rev. A 45, 1978 (1992); D. Delande and A. Buchleitner, Adv. At. Mol. Opt. Phys. 35, 85 (1994); I. BialynickiBirula, M. Kalinski, and J.H. Eberly, Phys. Rev. Lett. 73, 1777 (1994); A. Buchleitner and D. Delande, Phys. Rev. Lett. 75, 1487 (1995); M.V. Fedorov and S.M. Fedorov, Opt. Express 3, 271 (1998); M. Kalinski et al., Phys. Rev. A 67, 032503 (2003).

[8] H. Maeda and T.F. Gallagher, Phys. Rev. Lett. 92, 133004 (2004).

[9] L.G. Hanson and P. Lambropoulos, Phys. Rev. Lett. 74, 5009 (1995).

[10] X. Chen and J.A. Yeazell, Phys. Rev. Lett. 81, 5772 (1998).

[11] Absorption also plays a crucial role in the proposal [9] for and in the experiment [10] with an electronic nonspreading wave packet in a two-electron atom using atomic mode locking by loss modulation.

[12] For Michelangelo, sculpturing means "releasing the desired form from a block of marble by cutting away unwanted materials." See, for example, D. Preble, Artforms (Harper \& Row, New York, 1978).

[13] K.S. Johnson et al., Science 280, 1583 (1998); M.K. Oberthaler et al., Phys. Rev. A 60, 456 (1999); A. Turlapov et al., Phys. Rev. A 68, 023408 (2003).

[14] Throughout the paper we use $\hbar \equiv 1$.

[15] A. Scholz et al., Opt. Comm. 111, 155 (1994).

[16] M.A. Efremov et al., Laser Phys. 13, 995 (2003); M.V. Fedorov et al., JETP 97, 522 (2003). 
[17] This conclusion is only valid since our interferometric experiment discussed below exclude additional dynamics of the relative phases between diffraction orders.
[18] U. Drodofsky et al., Appl. Phys. B 65, 755 (1997); M. Mützel et al., Phys. Rev. Lett. 88, 083601 (2002). 